\title{
Case reports of invasive mucormycosis associated neutropenic enterocolitis in leukemic children: diagnostic and treatment challenges and review of literature
}

\author{
Ali Amanati ${ }^{*}$ (1) Omid Reza Zekavat ${ }^{2}$, Hamidreza Foroutan ${ }^{3}$, Omidreza Azh ${ }^{3}$, Ali Tadayon ${ }^{3}$, Ahmad Monabati ${ }^{4}$,
} Mohammad Hossein Anbardar ${ }^{5}$ and Haleh Bozorgi ${ }^{2}$

\begin{abstract}
Background: Bacterial enterocolitis is one of the most common neutropenic fever complications during intensive chemotherapy. Despite aggressive antibacterial treatments, this complication usually imposes high morbidity and mortality in cancer patients. Management of bacterial neutropenic enterocolitis are well known; however, management of fungal neutropenic enterocolitis may be more challenging and needs to be investigated. Prompt diagnosis and treatment may be life-saving, especially in patients at risk of mucormycosis-associated neutropenic enterocolitis.

Case presentation: We report two mucormycosis-associated neutropenic enterocolitis cases in pediatric leukemic patients receiving salvage chemotherapy for disease relapse. Both patients' clinical signs and symptoms differ from classical bacterial neutropenic enterocolitis. They were empirically treated as bacterial neutropenic enterocolitis with anti-gram-negative combination therapy. Despite broad-spectrum antimicrobial treatment, no clinical improvement was achieved, and both of them were complicated with severe abdominal pain necessitating surgical intervention. Mucormycosis is diagnosed by immunohistopathologic examination in multiple intraoperative intestinal tissue biopsies. Both patients died despite antifungal treatment with liposomal amphotericin-B and surgical intervention.

Conclusion: Mucormycosis-associated neutropenic enterocolitis is one of the most unfavorable and untreatable side effects of salvage chemotherapy in leukemic children with disease relapse. This report could be of considerable insight to the clinicians and scientists who counter the enigma of fungal infections during febrile neutropenia and help to understand better diagnosis and management.
\end{abstract}

Keywords: Mucormycosis, Leukemia, Neutropenic enterocolitis, Febrile neutropenia, Breakthrough fungal infection, Liposomal amphotericin B, Children

*Correspondence: ali_amanati_1356@yahoo.com; dr.amanati14@gmail.com; amanati@sums.ac.ir

1 Professor Alborzi Clinical Microbiology Research Center, Shiraz University of Medical Sciences, Shiraz, Islamic Republic of Iran

Full list of author information is available at the end of the article

\section{Background}

Neutropenic enterocolitis (NEC), also known as typhlitis, is a severe form of enterocolitis with an estimated incidence ranging from 1 to $10 \%$, varied based on age and underlying in disease neutropenic patients with malignancy [1-5]. The diagnosis is based on clinical, laboratory testing, and radiological assessment [6]. Fever, abdominal 
pain, nausea, vomiting, and diarrhea are common signs and symptoms in affected patients [7-9].

The most associated pathogens are gram-negative bacilli, gram-positive cocci, anaerobes, and rarely fungi $[6,7,10]$. Candida species are the most prevalent fungus associated with NEC, while Aspergillus has also been reported in a few cases [11]. Herein, we present unique fatal cases of invasive mucormycosis-associated neutropenic enterocolitis (IMANEC) in two leukemic children. Despite frequently reported gastrointestinal (GI) mucormycosis, confirmed IMANEC has never been reported to date in the context of febrile neutropenia in pediatric patients with leukemia.

\section{Case presentation \\ Case 1}

A 7-year-old girl, a known case of acute myeloblastic leukemia (AML), was admitted to our center due to central nervous system (CNS) relapse for reinduction chemotherapy on 7 October 2020. Her disease was diagnosed about 2-years before admission, and she was on maintenance chemotherapy. Reinduction chemotherapy started with cytarabine $\left(300 \mathrm{mg} / \mathrm{m}^{2}\right.$ as loading dose day 1 and 2 and continued by $150 \mathrm{mg} / \mathrm{m}^{2}$ from day 3), Etoposide, Idarubicin $(0.5 \mathrm{mg} / \mathrm{kg}$; day 3,5 , and 7$)$, and dexamethasone $1.5 \mathrm{mg} / 8 \mathrm{~h}$ (protocol BFM-2012). She also received trimethoprim/sulfamethoxazole $2.5 \mathrm{mg} / \mathrm{kg}$ twice/day three times/week to prevent Pneumocystis jirovecii pneumoniae (PJP) and antifungal prophylaxis with liposomal amphotericin-B (L-AmB) $2.5 \mathrm{mg} / \mathrm{kg}$ every other day concurrently.

After a week, her total white blood cell (WBC) count dropped to $1120 / \mathrm{mm}^{3}$ with absolute neutrophil count (ANC) $100 / \mathrm{mm}^{3}$, which continued to decrease to less than $50 / \mathrm{mm}^{3}$. She developed fever and abdominal pain predominantly in the right lower quadrant one week later. The diagnose of neutropenic enterocolitis (typhlitis) was made on 21 October, and she was treated with meropenem $(20 \mathrm{mg} / \mathrm{kg} /$ dose $/ 6 \mathrm{~h})$ and amikacin $(15 \mathrm{mg} / \mathrm{kg} /$ dose/ day), and the patient's diet changed to NPO (nil per os). Abdominopelvic ultrasonographic examination revealed a circumferentially thickened cecal and ascending colon (maximum diameter $3 \mathrm{~mm}$ ). The thickened colon walls were hyperechoic and hypervascular, surrounded by prominent lymph nodes and minimal free fluid, all together in favor of NEC. After four days, the patient's condition improved, and no thickened cecal and colon walls were detected in ultrasonographic examination (maximal thickness $1 \mathrm{~mm}$ ). A new episode of fever developed on 31 October. Sepsis workup was done, and meropenem switched to piperacillin-tazobactam. She also complicated Candida mucositis (WHO-grade 3), and her port-A-Cath and peripheral blood cultures became positive with non-albicans Candida (time to detection 15 and $16 \mathrm{~h}$, respectively). Systemic antifungal treatment started with caspofungin $\left(70 \mathrm{mg} / \mathrm{m}^{2}\right.$ loading dose and $50 \mathrm{mg} / \mathrm{m}^{2}$ as maintenance therapy), and the port-A-Cath was removed urgently. There was no evidence of fungal endocarditis in echocardiography. As follow patient again developed abdominal distention, bilious vomiting, and periumbilical abdominal pain. In the serial abdominopelvic ultrasonographic examination, a gradual increase in cecal and colon wall thickness was detected, and accordingly, the patient's diet changed to NPO.

She was referred to the surgical ward with suspicion of intestinal obstruction, and an exploratory laparotomy was performed. Intraoperative findings were matted small bowel with severe adhesion of omentum in the left upper quadrant with ischemic discoloration of $50 \mathrm{~cm}$ of small bowel after ligament Treitz along with multiple sites of yellowish discoloration of small bowel that started from ligament Treitz with skip lesions (1-5 cm diameter) separated them from each other along the $50 \mathrm{~cm}$ small bowel segment. Also, multiple yellowish lesions in the omentum and meso of the small bowel and transverse colon were found. Ladd's procedure (small bowel derotation) in addition to duodenal (2nd and 3rd part) and jejunal $(50 \mathrm{~cm})$ resection was done, and operation terminated with end-to-end duodenojejunostomy and drain insertion. The multiple biopsies were sent for pathology. $\mathrm{L}-\mathrm{AmB}$ ( $5 \mathrm{mg} / \mathrm{kg} /$ day) was started according to the intraoperative findings suggestive of fungal infection.

After a few days, she developed disseminated intravascular coagulation (DIC). Supportive intensive care unit (ICU) care in addition to broad-spectrum antibiotic coverage including vancomycin, colistin, and metronidazole and antifungal therapy with voriconazole was continued without significant clinical improvement. Finally, she died on 16 November 2020 with multi-organ failure. Multiple intestinal biopsies showed non-septate broad hyphae in the microscopic examination by hematoxylin and eosin (H\&E) staining (Fig. 1a-e). Several galactomannan tests were negative in her hospital course.

\section{Case 2}

A 9-year-old girl with acute lymphocytic leukemia (ALL) complicated with disease relapse after about 32 months of the induction chemotherapy was admitted with pancytopenia (total WBC count $1800 / \mathrm{mm}^{3}$, $\mathrm{Hb} 9.6 \mathrm{gr} / \mathrm{dL}$, and platelet $9000 / \mathrm{mm}^{3}$ ) on 20 November 2020. She received a reinduction regimen including cytarabine $\left(100 \mathrm{mg} / \mathrm{m}^{2}\right)$, Etoposide, weekly vincristine, and dexamethasone $2 \mathrm{mg} / 8 \mathrm{~h}$. She also received trimethoprim/sulfamethoxazole 200/40 mg twice/day three times/week for PJP prophylaxis and antifungal prophylaxis with L-AmB $2.5 \mathrm{mg} / \mathrm{kg}$ every other day 


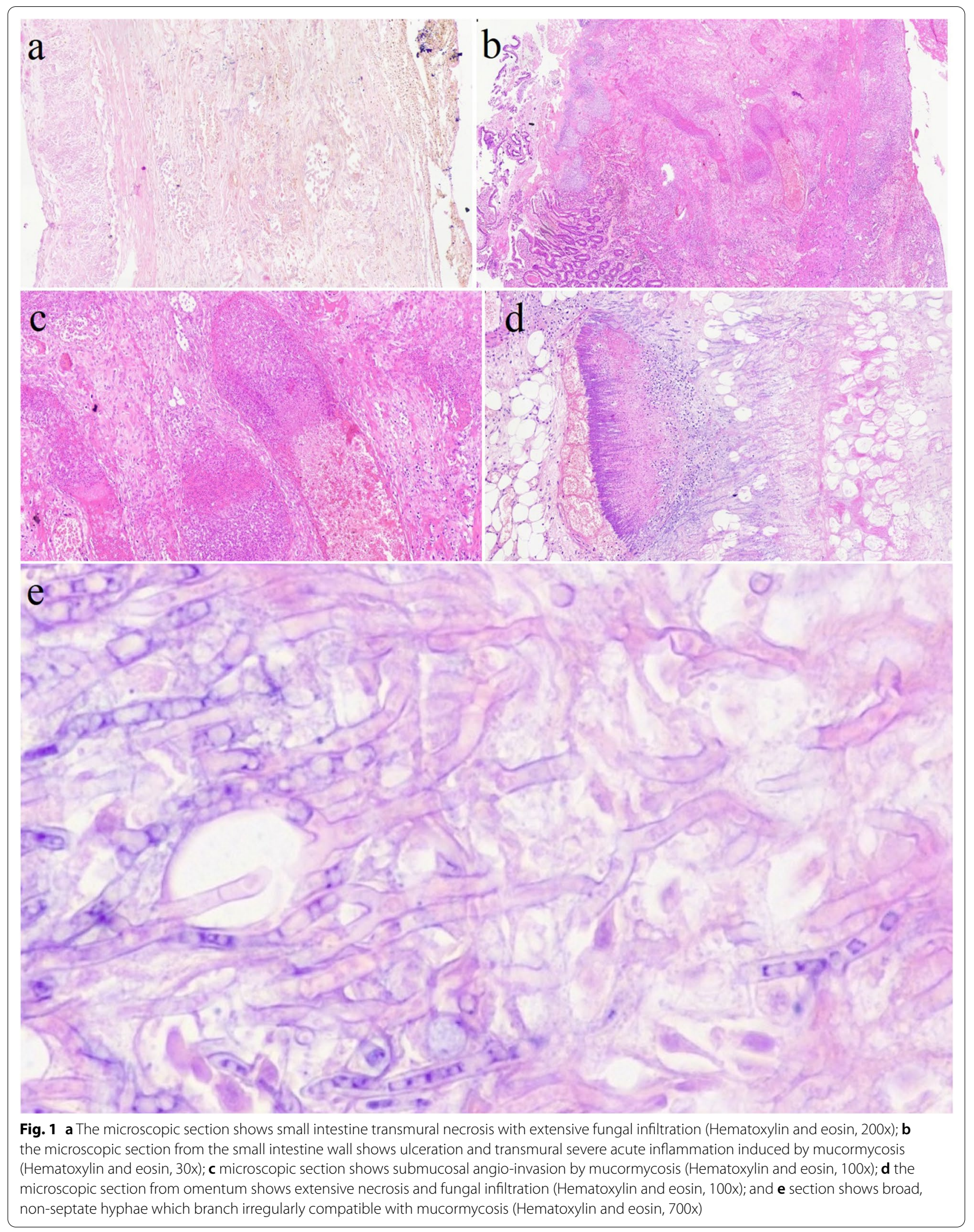


concurrent with her chemotherapy. During her admission course, she was neutropenic. She developed a fever on 30 November, when her WBC count dropped to $500 / \mathrm{mm}^{3}$. Full sepsis workup was done, and she was put on meropenem $(20 \mathrm{mg} / \mathrm{kg} /$ dose $/ 6 \mathrm{~h})$. Both port-A-Cath and blood peripheral culture became positive (time to detection 6 and $13 \mathrm{~h}$, respectively) with Klebsiella pneumoniae sensitive to meropenem. Both cultures reported negative after $72-\mathrm{h}$ of treatment.

A few days later, she complicated with abdominal pain after two weeks of profound neutropenia (absolute neutrophil count $<500 / \mathrm{mm}^{3}$ ). In the abdominopelvic ultrasonographic examination, the cecal wall was thickened with a maximum diameter of $2.38 \mathrm{~mm}$. The cecal wall was reported hyperechoic with mild hypervascularity. NEC was diagnosed, and the patient diet changed to NPO. Due to inadequate clinical response and continuous fever despite negative blood cultures, her antibiotic regimen changed to colistin $(150,000$ units $/ \mathrm{kg}$ as a loading dose then 75,000 units $/ \mathrm{kg} / 12 \mathrm{~h}$ ) and amikacin $(15 \mathrm{mg} / \mathrm{kg} /$ day $)$ on 5 December. The new antibiotic regimen achieved no clinical improvement, and with $\mathrm{L}-\mathrm{AmB}$ escalated to a therapeutic dose $(5 \mathrm{mg} / \mathrm{kg} /$ day $)$ after two days. Abdominopelvic ultrasonographic findings were worsened in serial examination (maximum cecal wall thickness about $3 \mathrm{~mm}$ ). In the third study, the small intestinal loops reveal increased thickness (maximum diameter $3.4 \mathrm{~mm}$ ) and ileocecal involvement. The patient's abdominal pain improved, and L-AmB continued, but she could not tolerate small liquid feeding. She was transferred to the pediatric surgery ward with suspicion of small bowel obstruction, suggestive of mucormycosis. Finally, she underwent exploratory laparotomy on 17 December. Post-operation findings were two lesions on $50 \mathrm{~cm}$ and $10 \mathrm{~cm}$ of ligament Treitz and adhesion band (Fig. 2). Appendectomy via double ligation method was done, and adhesiolysis and omental biopsy were taken and sent for pathology. After laparotomy, she developed DIC, which necessitated mechanical ventilation. Metronidazole $(300 \mathrm{mg} / 8 \mathrm{~h}$ ), linezolid (300 mg/12 h), and vitamin B6 (100 mg/day) were added to her antibiotic regimen, but she died on 22 December 2020 with multi-organ failure. In the pathology report, non-septate, broad hyphae were microscopic examinations of omental biopsy (Fig. $3 a-c$ ).

Mucormycosis was documented in intestinal tissue biopsies by fungal culture (Sabouraud dextrose agar; Merck, Germany), and non-septate broad hyphae were found on direct potassium hydroxide $(\mathrm{KOH})$ mount examination in both patients.

History of fresh carrot juice consumption was positive in both patients just before the clinical deterioration and worsening of the abdominal pain. It should be noticed

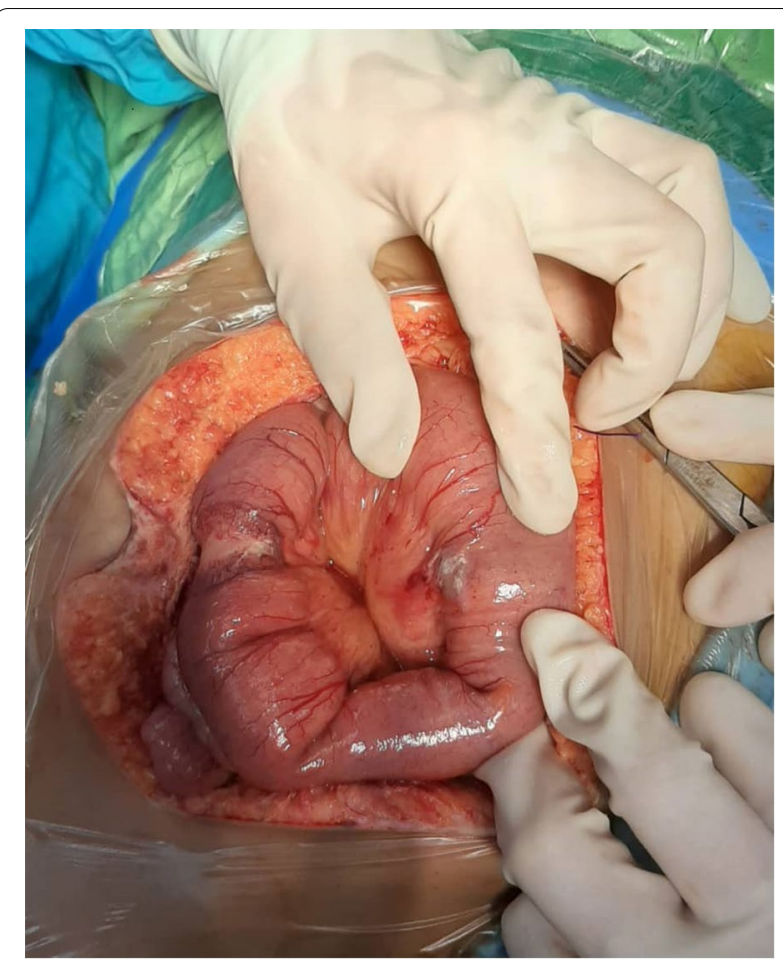

Fig. 2 After exploratory laparotomy, an adhesion band, was found over the intestinal loop. Adhesiolysis and resection of the stenosis segment of the small bowel were done, and the operation was finished by end-to-end anastomosis

that both of them only consume fresh carrot juice during this period.

Demographic characteristics and laboratory test results of two cases of invasive mucormycosis-associated neutropenic enterocolitis are summarized in Tables 1 and 2.

\section{Discussion and conclusions}

We provide a comprehensive review of two proven IMANEC in leukemic children with disease relapse. IMANEC is an uncommon presentation of invasive $\mathrm{Muc}$ orales infections [12], and there are very few reports of IMANEC in pediatric patients with hematological malignancies [13-15].

Vadeboncoeur et al. described a fatal small intestinal mucormycosis in a 31-month-old boy with large cell anaplastic lymphoma early after chemotherapy presented with a gangrenous ileo-ileal intussusception [16]. Sellappan et al. reported a colonic mucormycosis in a 10-year-old male child with Down's syndrome and B-precursor-ALL during induction chemotherapy with a fatal outcome [17]. Totadri et al. described a fatal small intestinal mucormycosis in a 10-year-old male child with B-precursor-ALL during the consolidation phase of chemotherapy [18]. 


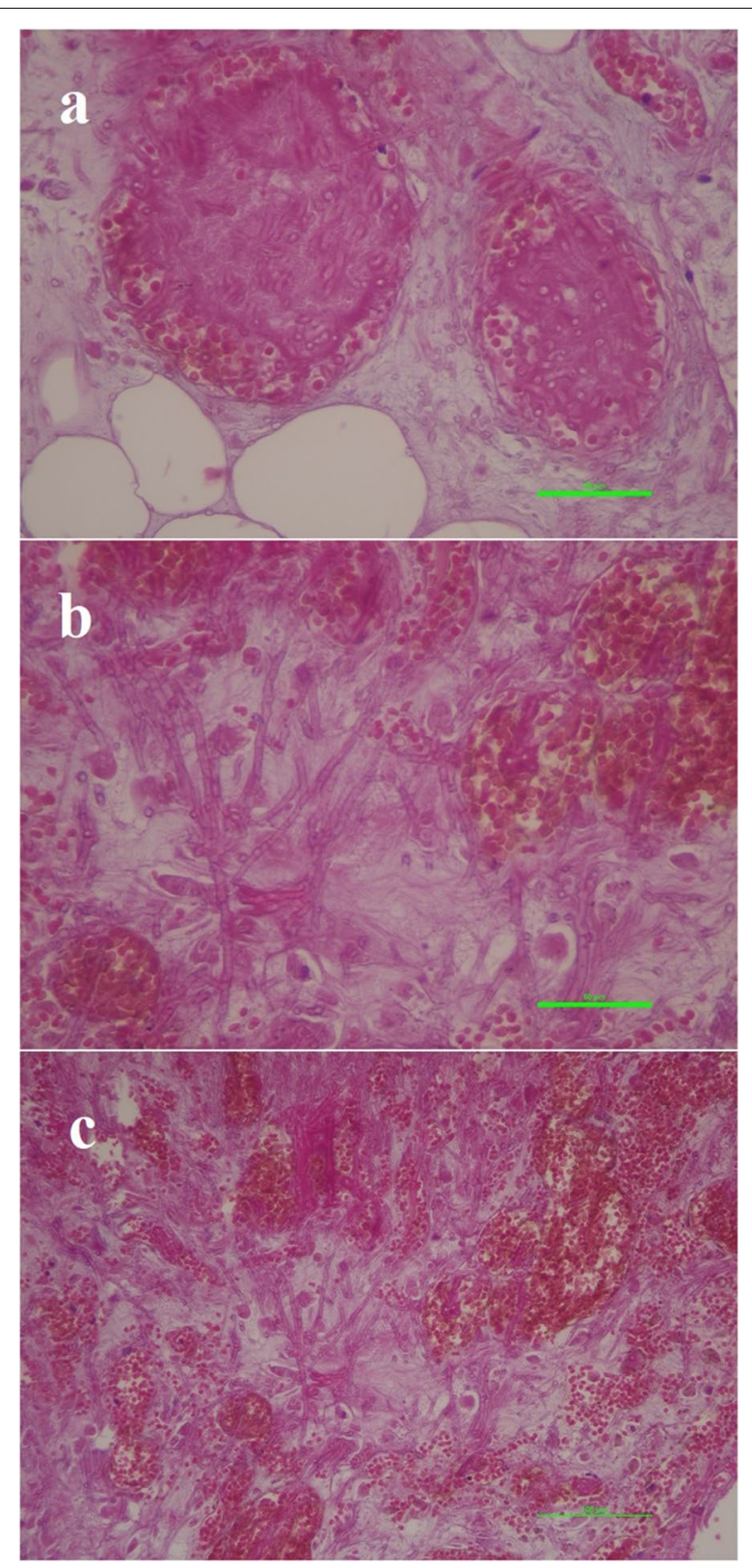

Fig. 3 a-c Sections of omental biopsy show fungal hyphae with no clear spore formation accompanied by inflammation and tissue congestion (scale bars represents $10 \mu \mathrm{m}, 50 \mu \mathrm{m}$, and $100 \mu \mathrm{m}$, respectively). H\&E X400. (images created by NIS-Elements software, Nikon Instruments Inc.)

So given the aggressive disease nature and its fatal outcome, this report could improve our knowledge regarding possible risk factors, clinical course, diagnostic and treatment challenges, and prognosis of mucormycosisassociated NEC.

The potential risk factors in our patients are summarized in Table 3. Identified risk factors for IMANEC could be categorized as host-related factors, medications, co-infections, comorbidities, and environmental exposure to fungal spores.

Host factors related to mucormycosis associated with neutropenic enterocolitis are malignancy type [19-21], disease station (relapse) $[19,21]$, prolonged neutropenia $[12,22]$, history of typhlitis, the intensity of chemotherapy-associated immunosuppression (including chemotherapy and corticosteroids) [12, 15, 23], unsolicited drug side effects (voriconazole and L-AmB prophylaxis) [23-25], coinfections [19, 26], trauma (including surgical interventions), tissue ischemia [15, 20], and contaminated food ingestion [21, 27]. Our patients have a history of fresh carrot juice consumption during the neutropenic phase. Although the patient's carrot juice samples were not accessible for us to test for fungal contamination, unpasteurized fruit juices usually have different levels of fungal contamination, including Rhizopus and Mucor [28].

\section{Diagnostic challenges}

Invasive mucormycosis usually is diagnosed by classical signs and symptoms representing specific organ involvement (for example, invasive rhinosinusitis [12, 27], cutaneous mucormycosis [12], and orbital involvement [29]). However, the diagnosis of mucormycosis is challenging due to the lack of standard mycological tests [21, 30], including circulating antigen detection tests and standard blood polymerase chain reaction (PCR) [31]. When prepared appropriately, biological tissue samples could confirm the diagnosis by culture and immunohistopathologic examination [32]. Repeated negative serum galactomannan (GM) tests may be used to support a diagnosis by rule outing invasive aspergillosis when clinical and radiological presentations do not help differentiate such mold infections [21]. Interventional diagnostic procedures usually are challenging to use because of bleeding tendency (including thrombocytopenia and coagulopathy) and profound neutropenia in vulnerable immunocompromised patients due to the risk of bleeding and secondary bacterial infections. Exploratory laparoscopy, endoscopy, and colonoscopy are often not applicable in patients suspected of GI mucormycosis. The main barriers to performing invasive diagnostic procedures are thrombocytopenia and the significant risk of postoperative intraabdominal complications. In this context, confirmed diagnosis usually is made in post-mortem autopsy examination of involved tissues.

Despite several limitations in the diagnostic approach of fungal associated NEC, a shifting pain (from right lower quadrant to other parts of the abdomen, especially contralateral quadrants), inadequate clinical response to broad-spectrum antibiotics, progressive 
Table 1 Summary characteristics of two cases of invasive mucormycosis-associated neutropenic enterocolitis in pediatric leukemia patients

\begin{tabular}{lll}
\hline & Case $\mathbf{1}$ & Case 2 \\
\hline Sex & Female & Female \\
Age & 7-year-old & 9-year-old \\
Underlying disease & Acute myeloid leukemia & Acute lymphoblastic leukemia \\
Disease station & Relapse (reinduction phase) & Relapse (reinduction phase) \\
Primary protocol & BMF-2012 & Standard risk ALL \\
Primary protocol date & October 2018 & April 2018 \\
Relapse protocol & BMF-2012 & MSK \\
Duration of neutropenia & 20 days & 17 days \\
AF prophylaxis & L-AmB & L-AmB \\
Duration of AF prophylaxis & 22 days & 10 days \\
FQ prophylaxis & Ciprofloxacin & Not used \\
Duration of FQ prophylaxis & 11 days & - \\
Primary involved site & Cecum & Cecum and ascending colon \\
First intestinal thickness & 2.9 mm & 2.38 mm \\
Dietary restriction period* & 27 days & 19 days \\
Blood culture & Non-albicans Candida & Klebsiella pneumonia \\
Concurrent Candida mucositis & WHO-III** & No \\
Empiric antimicrobial regimen & Meropenem plus amikacin & Colistin plus amikacin \\
History of typhlitis & Two times (December 2018) & No \\
History of infectious events & Perianal abscess, carbapenem-resistant gram-negative bactere- & No \\
& mia (Acinetobacter) &
\end{tabular}

$A F$ antifungal, $F Q$ fluoroquinolone

${ }^{*}$ Complete food and fluid withholding

${ }^{* *}$ World Health Organization-mucositis scale

ultrasonographic findings (increasing wall thickness or secondary small intestinal involvement), may be helpful clues for considering unusual etiologies such as mucormycosis for NEC. Fungal-associated NEC should be considered a breakthrough invasive fungal infection (bIFI) in neutropenic patients receiving antifungal prophylaxis such as L-AmB.

\section{Treatment}

The recommended strategy for treating invasive mucormycosis infections consists of anti-Mucorales antifungals, surgical debridement, and correcting predisposing risk factors [32]. Granulocyte colony-stimulating factor (G-CSF) and granulocyte-macrophage colony-stimulating factor (GMCSF) are commonly used for correct neutropenia [21]. Among available antifungals, L-AmB is one of the best anti-Mucorales due to lower nephrotoxicity and the possibility of administering higher drug doses, which could deliver higher cumulative drug concentrations in a shorter time [15]. However, in patients with breakthrough mucormycosis receiving L-AmB prophylaxis new azoles should be replaced, and antifungal class should be changed [15]. Posaconazole and isavuconazole are recommended antifungals [15, 33], but such formulations are usually not available in most medical, especially in developing countries.

\section{Breakthrough invasive fungal infection}

Although there is evidence that intermittent doses of L-AmB could prevent invasive fungal infections in immunocompromised hosts, some critical points should be considered. First, the effective prophylactic treatment with L-AmB is usually achieved by highdose intermittent intravenous injections [34-36], so the similar effect may not be obtained by lower doses as recommended by current guidelines for prophylaxis treatment $[37,38]$, which necessitates further investigation for determining optimal dosage and interval [39]. Second, the accumulation of the L-AmB varies according to the different body tissues during treatment [40]. Currently, intestinal tissue drug concentrations and the prophylactic effects of L-AmB for preventing invasive intestinal fungal infection are not evident. Third, the L-AmB accumulates inside the macrophage, and its effect diminishes when the fungus is invading and interacting extracellular matrix [41]. So, in food contamination, the prophylactic effects of L-AmB may be 
Table 2 Summary of laboratory test results of two cases of invasive mucormycosis-associated neutropenic enterocolitis

\begin{tabular}{|c|c|c|}
\hline & Case 1 & Case 2 \\
\hline \multicolumn{3}{|l|}{ At the time of typhlitis diagnosis } \\
\hline WBC count (per $\mathrm{mm}^{3}$ ) & 60 & 390 \\
\hline Hemoglobin (gr/dl) & 9 & 11.7 \\
\hline Platelet count (per mm³) & 80,000 & 93,000 \\
\hline Prothrombin time & - & 15.3 \\
\hline International normalized ratio & - & 1.06 \\
\hline Partial thromboplastin time & - & 27.9 \\
\hline Blood urea nitrogen (mg/dL) & 16 & 11 \\
\hline Serum creatinine (mg/dl) & 0.62 & 0.71 \\
\hline Alanine aminotransferase (U/L) & 30 & 69 \\
\hline Aspartate aminotransferase $(\mathrm{U} / \mathrm{L})$ & 16 & 46 \\
\hline Serum albumin & 3.8 & 3.4 \\
\hline ESR (mm/hours) & 125 & 111 \\
\hline CRP $(\mathrm{mg} / \mathrm{dL})$ & 23 & 41 \\
\hline \multicolumn{3}{|l|}{ On expiration date } \\
\hline WBC count (per mm³) & 1100 & 500 \\
\hline Hemoglobin (gr/dL) & 12.8 & 9.4 \\
\hline Platelet count (per mm³) & 17,000 & 17,000 \\
\hline Prothrombin time & 24.5 & 33.6 \\
\hline International normalized ratio & 1.81 & 2.49 \\
\hline Partial Thromboplastin Time & 59.1 & 39 \\
\hline Blood urea nitrogen (mg/dL) & 14 & 37 \\
\hline Serum creatinine (mg/dL) & 0.32 & 1.08 \\
\hline Alanine aminotransferase (U/L) & 14 & 49 \\
\hline Aspartate aminotransferase $(\mathrm{U} / \mathrm{L})$ & 23 & 82 \\
\hline Serum albumin & 3.5 & 3.2 \\
\hline $\mathrm{CRP}(\mathrm{mg} / \mathrm{dL})$ & $>150$ & $>150$ \\
\hline \multicolumn{3}{|l|}{ Final venous blood gas } \\
\hline $\mathrm{PH}$ & 7.25 & 7.018 \\
\hline CO2 concentration (mmHg) & 40.6 & 33 \\
\hline O2 concentration (mmHg) & 73.9 & 64 \\
\hline $\mathrm{HCO} 3(\mathrm{mmol} / \mathrm{L})$ & 17.9 & 8.5 \\
\hline Base excess (mmol/L) & -8.7 & -21.7 \\
\hline
\end{tabular}

WBC white blood cell, ESR Erythrocyte Sedimentation Rate, CRP C-reactive protein

* Both patients received fresh frozen plasma, vitamin-K, and packed cell several times

suboptimal compared with hematogenous intestinal involvement. Finally, the type of invasive fungus may predict the prophylactic effects of L-AmB. Although successful prophylaxis treatment with $\mathrm{L}-\mathrm{AmB}$ is expected for many fungus pathogens, similar effects for preventing invasive intestinal mucormycosis need to be investigated [42]. Altogether, mucormycosis-associated neutropenic enterocolitis may be considered a rare type of the bIFI in patients receiving L-AmB prophylaxis, as
Table 3 The potential identified risk factors in two cases of invasive mucormycosis-associated neutropenic enterocolitis

\section{Case 1}

Disease relapse

Prolonged neutropenia

History of recurrent typhlitis and recurrent Candida mucositis

Antifungal prophylaxis with L-AmB

Recent history of corticosteroid treatment

History of bacteremia (recent and past positive history)

History of fungemia

Natural carrot juice consumption early after the start of oral feeding

Case 2

Disease relapse

Prolonged neutropenia

Antifungal prophylaxis with L-AmB

Recent history of corticosteroid treatment

History of bacteremia

Natural carrot juice consumption early after the start of oral feeding

Local intestinal ischemia (adhesion band)

$L$-AmB liposomal amphotericin-B

we described in our previous reports in cancer patients [43].

\section{Prognosis and outcome}

Delayed proper antifungal therapy is the main prognostic factor in patients with invasive mucormycosis and substantially increased mortality [30, 44]. Besides, as documented by many researchers, antifungal therapy is usually accompanied by high mortality in invasive mucormycosis [32, 44]. Most of the reported surgical interventions are performed in patients with sino-orbital and cutaneous mucormycosis [32]. The prognosis of the GI mucormycosis and complicated NEC remained unknown due to small reported cases. As described earlier, IMANEC patients encountered several barriers to timely surgical interventions, so even timely antifungal treatment may lead to unfavorable outcomes in most cases.

In conclusion, it should be noticed that although a presumptive diagnosis of IMANEC usually is difficult, repeated negative GM tests, atypical predominant abdominal point tenderness or shifting pains, and inadequate clinical and ultrasonographic response may be helpful clues for considering unusual etiologies such as mucormycosis for NEC. Disease relapse, L-AmB prophylaxis, prolonged neutropenia, bacterial and fungal co-infections, natural fruit juice consumption may be potential risk factors for IMANEC in pediatric patients with leukemia. 


\begin{abstract}
Abbreviations
NEC: Neutropenic enterocolitis; IMANEC: Invasive mucormycosis-associated neutropenic enterocolitis; GI: Gastrointestinal; AML: Acute myeloblastic leukemia; CNS: Central nervous system; PJP: Pneumocystis jirovecii pneumoniae; L-AmB: Liposomal amphotericin-B; WBC: White blood cell; ANC: Absolute neutrophil count; DIC: Disseminated intravascular coagulation; ICU: Intensive care unit; ALL: Acute lymphocytic leukemia; PCR: Polymerase chain reaction; GM: Serum galactomannan tests; bIFl: Breakthrough invasive fungal infection; G-CSF: Granulocyte colony-stimulating factor; GMCSF: Granulocyte-macrophage colony-stimulating factor; ESR: Erythrocyte sedimentation rate; CRP: C-Reactive protein.

\section{Acknowledgements}

Our thanks go to Salma Mehrangiz (infection control unit staff), Somayeh Khajeh (microbiology department staff), infection control unit members in Amir medical oncology center, and Nemazee hospital PICU doctors and nurses for their kind's efforts and technical support.
\end{abstract}

\section{Authors' contributions}

The study concept and design: AA; Acquisition of data: AA, ORZ, HB; surgical interventions: HF, ORA, AT; Pathological reports: AM, MHA; Analysis and interpretation of data: $A A$; Drafting of the manuscript: $A A, H B$; Critical revision of the manuscript for important intellectual content: AA. All authors have read and approved the manuscript.

\section{Funding}

The authors have no support or funding to report.

\section{Availability of data and materials}

All data generated or analyzed during this study are included in this published article.

\section{Declarations}

\section{Ethics approval and consent to participate}

The ethics committee approved the study protocol at Professor Alborzi Clinical Microbiology Research Center, Shiraz University of Medical Sciences. The study protocol conformed to the ethical guidelines of the 1975 Helsinki Declaration.

\section{Consent for publication}

Written informed consent was obtained from the patients' parents to publish this case report and any accompanying images.

\section{Competing interests}

The authors declare that they have no competing interests.

\begin{abstract}
Author details
1Professor Alborzi Clinical Microbiology Research Center, Shiraz University of Medical Sciences, Shiraz, Islamic Republic of Iran. ${ }^{2}$ Hematology Research Center, Shiraz University of Medical Sciences, Shiraz, Islamic Republic of Iran. ${ }^{3}$ Laparoscopy Research Center, Shiraz University of Medical Sciences, Shiraz, Iran. ${ }^{4}$ Department of Hematopathology, Molecular Pathology and Cytogenetics, Shiraz University of Medical Sciences, Shiraz, Iran. ${ }^{5}$ Department of Pathology, School of Medicine, Shiraz University of Medical Sciences, Shiraz, Iran.
\end{abstract}

Received: 8 July 2021 Accepted: 7 December 2021

Published online: 20 December 2021

\section{References}

1. Fike FB, Mortellaro V, Juang D, Peter SDS, Andrews WS, Snyder CL. Neutropenic colitis in children. J Surg Res. 2011;170(1):73-6.

2. Rodrigues FG, Dasilva G, Wexner SD. Neutropenic enterocolitis. World J Gastroenterol. 2017;23(1):42.

3. Gorschlüter M, Mey U, Strehl J, Ziske C, Schepke M, Schmidt-Wolf IG, Sauerbruch T, Glasmacher A. Neutropenic enterocolitis in adults: systematic analysis of evidence quality. Eur J Haematol. 2005;75(1):1-13.

4. Cartoni C, Dragoni F, Micozzi A, Pescarmona E, Mecarocci S, Chirletti P, Petti MC, Meloni G, Mandelli F. Neutropenic enterocolitis in patients with acute leukemia: prognostic significance of bowel wall thickening detected by ultrasonography. J Clin Oncol. 2001;19(3):756-61.

5. Dorantes-Díaz D, Garza-Sánchez J, Cancino-López J, Rocha-Ramírez J, Rojas-Illanes F, Parrado-Montaño J, Hernández-Escalante V. Prevalence of neutropenic enterocolitis in adults with severe neutropenia and associated mortality. Rev Gastroenterol Mex. 2009;74(3):224-9.

6. Nesher L, Rolston KV. Neutropenic enterocolitis, a growing concern in the era of widespread use of aggressive chemotherapy. Clin Infect Dis. 2013;56(5):711-7.

7. Sachak T, Arnold MA, Naini BV, Graham RP, Shah SS, Cruise M, Park JY, Clark L, Lamps L, Frankel WL. Neutropenic enterocolitis. Am J Surg Pathol. 2015;39(12):1635-42.

8. Machado NO. Neutropenic enterocolitis: a continuing medical and surgical challenge. N Am J Med Sci. 2010;2(7):293.

9. Cloutier RL. Neutropenic enterocolitis. Hematol Oncol Clin North Am. 2010;24(3):577-84.

10. Song L, Marcon NE. Neutropenic enterocolitis (typhlitis). UpToDate, Waltham, MA 2017.

11. Gorschlüter M, Mey U, Strehl J, Schmitz V, Rabe C, Pauls K, Ziske C, Schmidt-Wolf IG, Glasmacher A. Invasive fungal infections in neutropenic enterocolitis: a systematic analysis of pathogens, incidence, treatment and mortality in adult patients. BMC Infect Dis. 2006;6:35.

12. Serris A, Danion F, Lanternier F. Disease entities in mucormycosis. J Fungi. 2019;5(1):23. https://doi.org/10.3390/jof5010023.

13. Lyon DT, Schubert TT, Mantia AG, Kaplan MH. Phycomycosis of the gastrointestinal tract. Am J Gastroenterol. 1979;72(4):379-94.

14. Mooney JE, Wanger A. Mucormycosis of the gastrointestinal tract in children: report of a case and review of the literature. Pediatr Infect Dis J. 1993;12(10):872-6.

15. Cornely OA, Alastruey-Izquierdo A, Arenz D, Chen SC, Dannaoui E, Hochhegger B, Hoenigl M, Jensen HE, Lagrou K, Lewis RE. Global guideline for the diagnosis and management of mucormycosis: an initiative of the European Confederation of Medical Mycology in cooperation with the Mycoses Study Group Education and Research Consortium. Lancet Infect Dis. 2019;19(12):e405-21.

16. Vadeboncoeur C, Walton J, Raisen J, Soucy P, Lau H, Rubin S. Gastrointestinal mucormycosis causing an acute abdomen in the immunocompromised pediatric patient—-three cases. J Pediatr Surg. 1994;29(9):1248-9.

17. Sellappan B, Bakhshi S, Safaya R, Gupta AK, Arya LS. Invasive colonic mucormycosis in early induction therapy of childhood acute lymphoblastic leukemia. Indian J Pediatr. 2005;72(1):77-9.

18. Totadri S, Sundersingh S, Natarajan R, Seshadri R, Radhakrishnan V. Gastrointestinal mucormycosis in a child with acute lymphoblastic leukemia: an uncommon but ominous complication. Indian J Cancer. 2018;55(3):304-5.

19. Stanzani M, Lewis RE, Fiacchini M, Ricci P, Tumietto F, Viale P, Ambretti S, Baccarani M, Cavo M, Vianelli N. A risk prediction score for invasive mold disease in patients with hematological malignancies. PLOS ONE. 2013;8(9): e75531.

20. Ambrosioni J, Bouchuiguir-Wafa K, Garbino J. Emerging invasive zygomycosis in a tertiary care center: epidemiology and associated risk factors. Int J Infect Dis. 2010;14:e100-3.

21. Kontoyiannis DP, Lewis RE. How I treat mucormycosis. Blood J Am Soc Hematol. 2011;118(5):1216-24.

22. Lien M-Y, Chou C-H, Lin C-C, Bai L-Y, Chiu C-F, Yeh S-P, Ho M-W. Epidemiology and risk factors for invasive fungal infections during induction chemotherapy for newly diagnosed acute myeloid leukemia: a retrospective cohort study. PLoS ONE. 2018;13(6): e0197851.

23. Singh N, Aguado JM, Bonatti H, Forrest G, Gupta KL, Safdar N, John GT, Pursell KJ, Muñoz P, Patel R. Zygomycosis in solid organ transplant recipients: a prospective, matched case-control study to assess risks for disease and outcome. J Infect Dis. 2009;200(6):1002-11.

24. Reid G, Lynch JP III, Fishbein MC, Clark NM. Mucormycosis. Semin Respir Crit Care Med. 2020;41:099-114. https://doi.org/10.1055/s-0039-3401992.

25. Perrella A, Esposito C, Amato G, Perrella O, Migliaccio C, Pisaniello D, Calise F, Cuomo O, Santaniello W. Antifungal prophylaxis with liposomal amphotericin B and caspofungin in high-risk patients after liver transplantation: impact on fungal infections and immune system. Infect Dis. 2016;48(2):161-6.

26. Makino K, Aoi J, Egashira S, Honda N, Kubo Y, Kawakami Y, Hayashi H, Mochizuki T. Multiple skin abscesses caused by Rhizopus sp. infection 
after Candida albicans infection in an immunocompromised patient. Med Mycol J. 2019;60(1):17-21.

27. Tournas V, Heeres J, Burgess L. Moulds and yeasts in fruit salads and fruit juices. Food Microbiol. 2006;23(7):684-8.

28. Moss M. Fungi, quality and safety issues in fresh fruits and vegetables. J Appl Microbiol. 2008;104(5):1239-43.

29. Amanati A, Barzegar H, Pouladfar G, Dashti AS, Abtahi MB, Khademi B, Ashraf MJ, Badiee P, Hamzavi SS, Kashkooe A. Orbital mucormycosis in immunocompetent children; review of risk factors, diagnosis, and treatment approach. BMC Infect Dis. 2020;20(1):1-9.

30. Chamilos G, Lewis RE, Kontoyiannis DP. Delaying amphotericin B-based frontline therapy significantly increases mortality among patients with hematologic malignancy who have zygomycosis. Clin Infect Dis. 2008;47(4):503-9.

31. Donnelly JP, Chen SC, Kauffman CA, Steinbach WJ, Baddley JW, Verweij PE, Clancy CJ, Wingard JR, Lockhart SR, Groll AH. Revision and update of the consensus definitions of invasive fungal disease from the European Organization for Research and Treatment of Cancer and the Mycoses Study Group Education and Research Consortium. Clin Infect Dis. 2020;71(6):1367-76.

32. Cornely O, Arikan-Akdagli S, Dannaoui E, Groll A, Lagrou K, Chakrabarti A, Lanternier F, Pagano L, Skiada A, Akova M. ESCMID and ECMM joint clinical guidelines for the diagnosis and management of mucormycosis 2013. Clin Microbiol Infect. 2014:20:5-26.

33. Maschmeyer G, Patterson TF. Our 2014 approach to breakthrough invasive fungal infections. Mycoses. 2014;57(11):645-51.

34. Mehta P, Vinks A, Filipovich A, Vaughn G, Fearing D, Sper C, Davies S. High-dose weekly Am Bisome antifungal prophylaxis in pediatric patients undergoing hematopoietic stem cell transplantation: a pharmacokinetic study. Biol Blood Marrow Transplant. 2006;12(2):235-40.

35. Annino L, Chierichini A, Anaclerico B, Finolezzi E, Norata M, Cortese S, Cassetta MI, Fallani S, Novelli A, Girmenia C. Prospective phase II single-center study of the safety of a single very high dose of liposomal amphotericin $B$ for antifungal prophylaxis in patients with acute myeloid leukemia. Antimicrob Agents Chemother. 2013;57(6):2596-602.

36. Hand EO, Ramanathan MR. Safety and tolerability of high-dose weekly liposomal amphotericin B antifungal prophylaxis. Pediatr Infect Dis J. 2014:33(8):835-6.

37. Lehrnbecher T, Fisher BT, Phillips B, Beauchemin M, Carlesse F, Castagnola E, Duong N, Dupuis LL, Fioravantti V, Groll AH. Clinical practice guideline for systemic antifungal prophylaxis in pediatric patients with cancer and hematopoietic stem-cell transplantation recipients. J Clin Oncol. 2020;38(27):3205.

38. Ullmann AJ, Aguado JM, Arikan-Akdagli S, Denning DW, Groll AH, Lagrou K, Lass-Flörl C, Lewis RE, Munoz P, Verweij PE. Diagnosis and management of Aspergillus diseases: executive summary of the 2017 ESCMID-ECMMERS guideline. Clin Microbiol Infect. 2018;24:e1-38.

39. Schiave LA, Nascimento E, Gaspar GG, Vilar FC, Martinez EZ. Gaitani CMd, Martinez R: Minimum concentration of Amphotericin B in serum according to the formulation, dose, and daily or prolonged intermittent therapeutic regimen. Rev Soc Bras Med Trop. 2020. https://doi.org/10. 1590/0037-8682-0463-2018.

40. Smith PJ, Olson JA, Constable D, Schwartz J, Proffitt RT, Adler-Moore JP. Effects of dosing regimen on accumulation, retention and prophylactic efficacy of liposomal amphotericin B. J Antimicrob Chemother. 2007;59(5):941-51.

41. Ibrahim AS. Host cell invasion in mucormycosis: role of iron. Curr Opin Microbiol. 2011;14(4):406-11.

42. Ellis M. New dosing strategies for liposomal amphotericin B in high-risk patients. Clin Microbiol Infect. 2008;14:55-64.

43. Amanati A, Lotfi M, Masoudi MS, Jafarian H, Ghasemi F, Bozorgi $H$, Badiee P. Cerebral and pulmonary aspergillosis, treatment and diagnostic challenges of mixed breakthrough invasive fungal infections: case report study. BMC Infect Dis. 2020;20(1):1-7.

44. Brunet K, Rammaert B. Mucormycosis treatment: Recommendations, latest advances, and perspectives. J Mycol Méd. 2020;30(3): 101007.

\section{Publisher's Note}

Springer Nature remains neutral with regard to jurisdictional claims in published maps and institutional affiliations.

Ready to submit your research? Choose BMC and benefit from:

- fast, convenient online submission

- thorough peer review by experienced researchers in your field

- rapid publication on acceptance

- support for research data, including large and complex data types

- gold Open Access which fosters wider collaboration and increased citations

- maximum visibility for your research: over $100 \mathrm{M}$ website views per year

At BMC, research is always in progress.

Learn more biomedcentral.com/submissions 